\title{
Review of: "Tropical Terrestrial And Epiphytic Ferns Have Different Leaf Stoichiometry With Ecological Implications."
}

\author{
Wen-Liang Chiou
}

Potential competing interests: The author(s) declared that no potential competing interests exist.

\section{General comments:}

Authors investigated the concentrations of folia elements (N, P, K, Ca and Mg) in three tropical forests of Brunei Darussalam. That aims to elucidate the stoichiometry relationship between terrestrials (16 species) and epiphytes (4 species) and between species of two evolutionary clades, i.e., polypoid and nonpolypod. Results bring abundant information of the stoichiometry distribution on those studied tropical Asian fern species. The meanings of those data on the ecophysiology and adaptation of those species are also discussed. These results provide a valuable basis to assess the potential risk of those species under different types of habitats due to the disturbance or climate change. However, due to some unclear (and confusing) definitions and insufficient analyses and descriptions, which may cause inappropriate conclusion, this manuscript is suggest to be MAJORLY revised before accepted to be published.

Specific comments:

1. Stoichiometry comparison between polypoid and non-polypod clades is one of the main issues of this study. It is encouraged to address more regarding the relationship of the stoichiometry and evolution (phylogeny), not only comparing higher/lower concentration/ratio of the said elements.

2. Many stoichiometry element ratios are shown in the results, but many of them, such as N:Ca, N:Mg, P:K, $\mathrm{P}: \mathrm{Ca}, \mathrm{P}: \mathrm{Mg}$, etc. are not well illustrated their relevance on ecophysiology or adaptation to habitats.

3. Sampling numbers of epiphytes (4 species) are very lower than of terrestrials ( 16 species). This bias would distort the general conclusion. For example, the high Ca concentration in two epiphytic species results in the significant difference between epiphytes and terrestrials although the other two epiphytes do not have such high Ca concentration. In fact, all elements face the same situation in this study when compare these two lifeforms. When the sampling number is small, overweighting is prone to occur and the conclusion needs to be more conservative or at least remind audiences this bias sampling and possible distortion.

4. In DISCUSSION (P.9), it mentions that the high concentration of $\mathrm{Ca}$ and $\mathrm{K}$ is likely that epiphytic species experience frequent and long periods of drought in an epiphytic habitat which promotes effectively taking up $\mathrm{K}$. The region of this study is under humidity of approximately $82 \%$ and with annual precipitation of $2,722 \mathrm{~mm}$ (P.4). It seems that this region is not drought. In addition, in dipteroid forest, 
it is well known that nutrient is poor in soil but rich in trunk. Therefore the element absorption from bark is not impossible. However, the nutritional status of epiphytic hosts was not assessed (see METHODS (P.4).

5. The most confusing thing is the definition of "ratio" and "concentration". The latter should have unit ( $\mathrm{mg} / \mathrm{g}$, in this case) but the former should have not. In this manuscript, the unit $(\mathrm{mg} / \mathrm{g}$ ) often comes with ratio (Table 1, 2 and many descriptions of the text). On the other hand, when mentioning concentration, it did not clear point on which element (see Tables 1 and 2). It must be clarified.

6. Other comments/suggestions are marked and noted in the attached pdf file. 